\title{
Modeling and Control of Airport Queueing Dynamics under Severe Flow Restrictions
}

\author{
Francis Carr \\ fcarr@mit .edu
}

\author{
Antony Evans \\ antonye@mit .edu
}

\author{
John-Paul Clarke \\ johnpaulomit .edu
}

\author{
Eric Feron \\ feronomit.edu
}

\author{
International Center for Air Transportation \\ Massachusetts Institute of Technology \\ Cambridge MA 02139
}

\begin{abstract}
Based on field observations and interviews with controllers at BOS and EWR, we identify the closure of local departure fixes as the most severe class of airport departure restrictions. A set of simple queueing dynamics and "traffic rules" are developed to model departure traffic under such restrictions. The validity of the proposed model is tested via Monte Carlo simulation against 10 hours of actual operations data collected during a case-study at EWR on June 29,2000 . In general, the model successfully reproduces the aggregate departure congestion. An analysis of the average error over 40 simulation runs indicates that flow-rate restrictions also significantly impact departure traffic; work is underway to capture these effects. Several applications and "what-if" scenarios are discussed for future evaluation using the calibrated model.
\end{abstract}

\section{Introduction}

A substantial fraction of the delays and uncertainties in gateto-gate block time manifest during the taxi-out process, between the gates and the departure runways. Although slowdowns in the taxi-out process can often be traced to local traffic conditions such as excessive departure demand, previous research at Boston-Logan Airport (BOS) has indicated that restrictions imposed by downstream airspace and airports can also create artificial local bottlenecks, leading to local traffic congestion and delays [7, (chap. 3)]. Thus, it is important to consider the effects of downstream restrictions when attempting to analyze, model, and optimize departure traffic flows on the airport surface. In this paper, we extend previous research on modeling and control of the airport departure process $[2,3,5,6,9,12]$ to incorporate the effects of downstream traffic restrictions.

Restrictions may generally be divided into flow-rate limits [e.g. Miles-In-Trail (MIT) or MINutes-In-Trail (MINIT) spacing requirements through a departure fix] or timewindowing for specific aircraft (e.g. Ground Delay Pro- grams to congested airports). Note that both types of restrictions have an effective cutoff threshold, above which traffic is stopped completely. Restrictions can also be categorized by spatial scope:

- Local airspace such as departure fixes;

- Downstream airspace such as neighboring sectors;

- Downstream airports;

and temporal scope:

- Lead-time at which changes to restrictions may be predicted accurately;

- Expected duration of a given restriction.

This paper focuses on successfully modeling the most significant ("first-order") effects of downstream restrictions, where the relative severity is measured in terms of overall impact on aggregate metrics such as airport throughput, departure congestion, and average taxi-out delay.

\section{Fieldwork and Data Sources}

In [7, (chap. 3)], it was observed that flow-rate restrictions caused by local weather at the airport's departure fixes tended to produce the greatest deviations from average throughput and thus the greatest delay. Interviews with air traffic controllers at BOS tower and Terminal Radar Approach Control (TRACON) support this observation:

1. Non-local restrictions impact a much smaller fraction of traffic than local restrictions. For example, arrival restrictions to a given downstream airport may cause flights to be cancelled outright (achieving the maximum impact on throughput and delays for that particular flight), but relatively few fights are destined for any particular airport. 
2. Time-windowing has a smaller impact than flowrestrictions. Again, time-windowing is typically imposed to merge a flight into an available traffic slot (either enroute or at the destination airport), and thus affects a smaller fraction of the total departure traffic.

3. Up to a certain threshold of severity, controllers ${ }^{1}$ can usually accommodate spacing restrictions at a departure fix (either MIT or MINIT) via proper sequencing of the runway queues and vectoring in the TRACON airspace before handoff to the Center. This flexibility is primarily limited by the workload involved in sequencing and the mix of traffic among the various departure fixes. All of the controllers interviewed said that closing a departure fix had a much greater effect than the spacing restrictions normally encountered, which range from 2 to 8 times the normal minimum en-route spacing.

4. Small spacing requirements at a departure fix (e.g. stretching the minimum 3-mile takeoff spacing to the minimum 5-mile enroute spacing) can be accomplished in the TRACON airspace; however, there are workload issues involved with the necessary aircraft vectoring. Large spacing requirements (especially MINIT restrictions) can and must be imposed at the runway.

Based on these observations, this paper focuses primarily on the effects of closing one or more local departure fixes.

A very important factor in this research is the availability of a rich set of operations data from the Newark Airport (EWR) case-study [4]. Operations data from the CATER system at EWR provide a detailed record of traffic demand, including runway configurations and epochs for pushbackclearance request and takeoff. Restriction logs from EWR tower and photocopied flight strips are also available, and provide a reliable recording of the traffic situation handled by the controllers.

As shown in Figure 1, EWR has a primary pair of dependent runways oriented in a NE/SW configuration ( $4 / 22 \mathrm{R} / \mathrm{L}$ ), and a secondary east/west runway (11/29). According to an interview with the tower supervisor, $11 / 29$ is used only infrequently; analysis of the CATER data shows less than $4 \%$ of the recorded traffic using 11/29. Typically, arrivals are shunted to the outer runway of the pair, while departures are taken off the inner runway. This is primarily due to limited taxiway space, both for departure queues between the runways, and for arrival queues between the inner runway and the terminal buildings. Due to these facts, departure traffic at EWR has a simple flow-pattern, with only a single potential runway queue in each of the two possible runway configurations.

\footnotetext{
${ }^{1}$ Specifically, the Ground and Local positions in the tower, and the Departure position(s) in the TRACON.
}

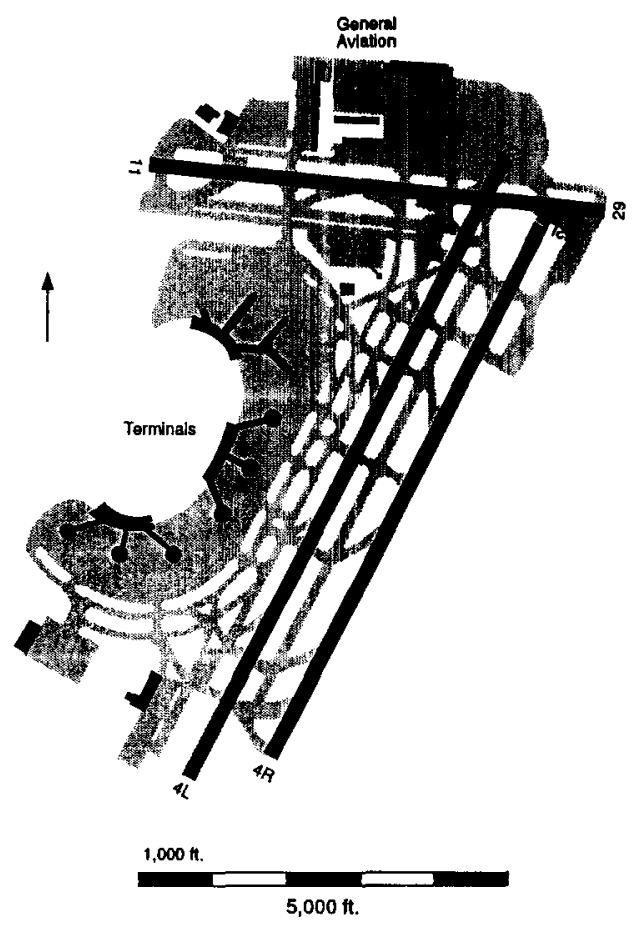

Figure 1: Runway layout at EWR.

\section{Model Dynamics}

A very large number of factors can influence the taxi-out time of any given flight; very few of these factors are presently recorded in the available operations data. For this reason, the traffic dynamics we propose are intended to capture the average input-output behavior of the airport departure process rather than the detailed motion of individual aircraft across the airport surface. Factors which are not currently recorded in the available operations data are lumped together and represented as stochastic effects.

Aircraft are assumed to enter the system when the pilot requests clearance to taxi. If a given aircraft is not subject to any downstream flow restrictions, the following dynamics based on [3, 9] are used (see the right-hand side of Figure 2). The taxi clearance is assumed to be delivered immediately. After receiving clearance, the aircraft undergoes a stochastic nominal taxi-out time based on its origin on the airport surface. Good approximations for the "origin" of a departing flight include its gate, terminal, or airline (since airlines typically cluster their gates physically). Factors such as communications delay with the tower, lack of proper weight-and-balance numbers, and interactions with other taxiing aircraft are not observed in current operations data and thus must be lumped into the stochastic nominal taxi-out time. For purposes of model calibration, probability distributions for these taxi-out times are estimated from the relatively unimpeded taxi-out times observed at low departure congestion levels. 


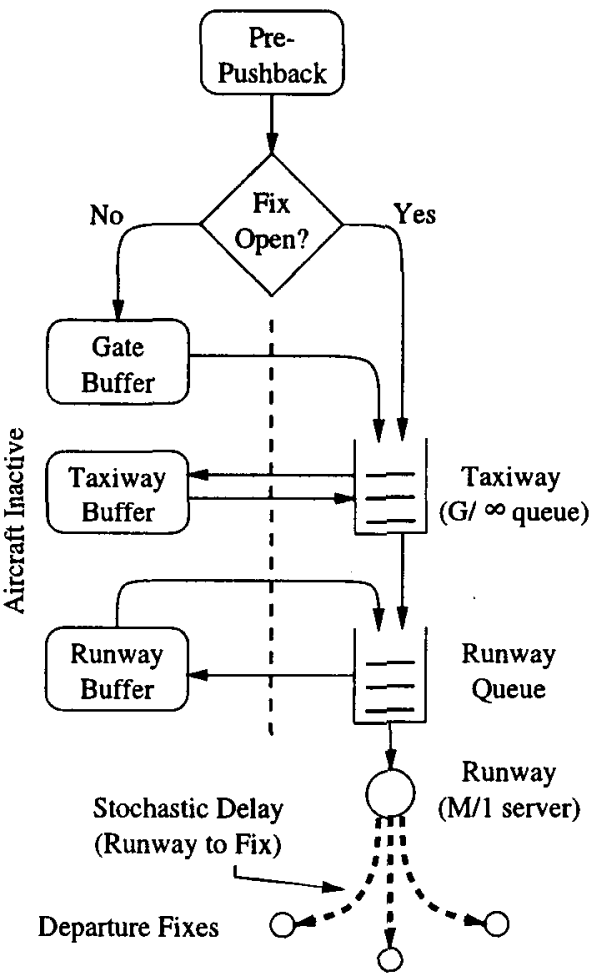

Figure 2: Queueing model dynamics.

Once the (unrestricted) aircraft completes its stochastic nominal taxi-out time, it enters a FCFS queue, which represents the departure queues typically observed near airport runways [8]. The aircraft is then assigned a stochastic runway service-time. For purposes of model calibration; the runway service-rate is estimated during periods of high departure congestion when the runway is operating at maximum throughput with high probability. After working its way through the queue and being serviced at the runway, the aircraft leaves the system.

A flight whose departure fix has only a finite spacing requirement is subject to the queueing dynamics described previously. It is assumed that tower and TRACON controllers have sufficient "control bandwidth" to meet the spacing requirement, either through sequencing at the runway or vectoring in the departure airspace. However, when a fix is completely closed, the affected flights are subject to a modified set of rules (see the left-hand side of Figure 2):

1. Affected flights which have not yet received clearance to taxi are held until the fix closure is lifted.

2. Affected flights which are taxiing out to the runway are pulled into "penalty boxes" or into temporary parking areas on the airport surface. The duration of the fix-closure is added to their stochastic nominal taxi-out time. Essentially, affected aircraft cannot make progress towards the runway, but also do not "lose ground" by being sent back to the gates.
3. Affected flights in the runway queue are similarly pulled out of the queue and held in a staging area. However, when the fix closure is lifted, the affected aircraft join the end of the runway queue; their previous position in the queue is not reserved or saved.

These rules have been developed from observations made while studying EWR, and are intended to mimic the actual airline and air-traffic control strategies observed during two days of very high delays and heavy departure fix restrictions.

It is important to note that the current model dynamics do not include the effect of finite parking space on the airport surface. Lack of sufficient buffering space can severely constrain airport surface traffic. For example, during recent site-visits with airline station managers at BOS, it was often noted that bottlenecks due to overloaded ramp buffers can cause major traffic foul-ups. However, during the casestudy it was observed that under conditions of extreme congestion, much (sometimes most) of the EWR airfield could be converted into parking space for delayed flights [4]. Hence we have not included the effects of finite buffering space in the current model dynamics.

\section{Comparison of Simulated and Observed Operations}

\subsection{Simulation Inputs}

The queueing dynamics described above have been coded into a Matlab-based Monte Carlo simulation. The downstream restrictions affecting EWR were obtained from TRACON logs covering 11:00 to 19:00 (EDT) on June 29, 2000 ; the results discussed below focus on this period. The reported statistics are aggregated from 40 simulation runs, each covering the same 8 -hour period and driven by identical inputs. These inputs included the actual fix assignments and pushback demand (taken from the flight-strips), and a time-history of the status of each fix (open or closed).

The runway service rate is approximated with a triangular density to match the service rate observed during the first 2 hours of lightly-restricted traffic on June 29, 2000:

$$
f(x \text { minutes })=\left\{\begin{array}{cc}
x, & 0<x<1 \\
2-x, & 1<x<2
\end{array}\right.
$$

Probability distributions for the nominal stochastic taxi-out times cannot be calibrated using the two days covered by the case-study due to the abnormal delays. Instead, nominal taxi-out times for the major passenger airlines at EWR have been calibrated from the 1998 Airline Service Quality Performance (ASQP) database using the techniques described in [3]. Service-times for air carriers not represented in ASQP have been approximated using a normal distribution, with mean and variance equal to the statistics measured for ASQP airlines. 


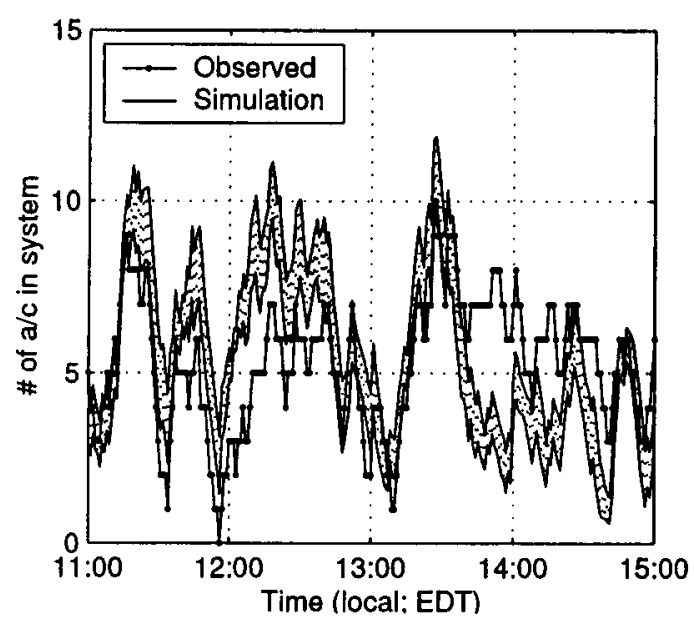

Figure 3: Number of departing a/c (early).

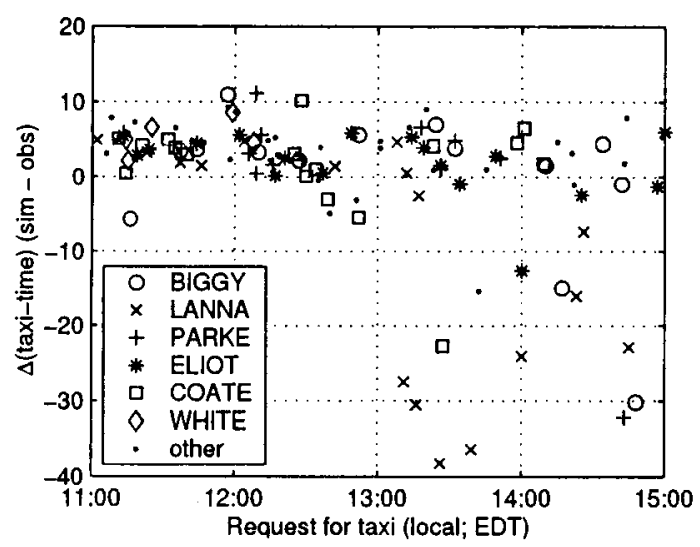

Figure 4: Simulation error (early).

\subsection{Simulation Results}

As shown in Figure 3, the model dynamics accurately reproduce the actual number of departing aircraft in the system over the (relatively unrestricted) period from 11:00 to 15:00. The vertical axis plots the number of departing aircraft on the airport surface. The solid line with dots is the actual observed number, while the thick "meshed" line represents the simulation aggregate (average plus/minus one standard deviation).

For each flight, we define the simulation error as the difference between average simulated and actual takeoff times. Figure 4 plots the simulation error for each flight against its pushback time. 107 of the 122 flights which pushed back during this period have an error of 10 minutes or less $(88 \%)$, and 8 of the outliers are using the LANNA fix. It is hypothesized that the LANNA outliers can be partially explained by the 40MIT restriction on LANNA from 13:05 to 15:50, and more work is necessary to refine the model dynamics and include restrictions other than outright fix closures.

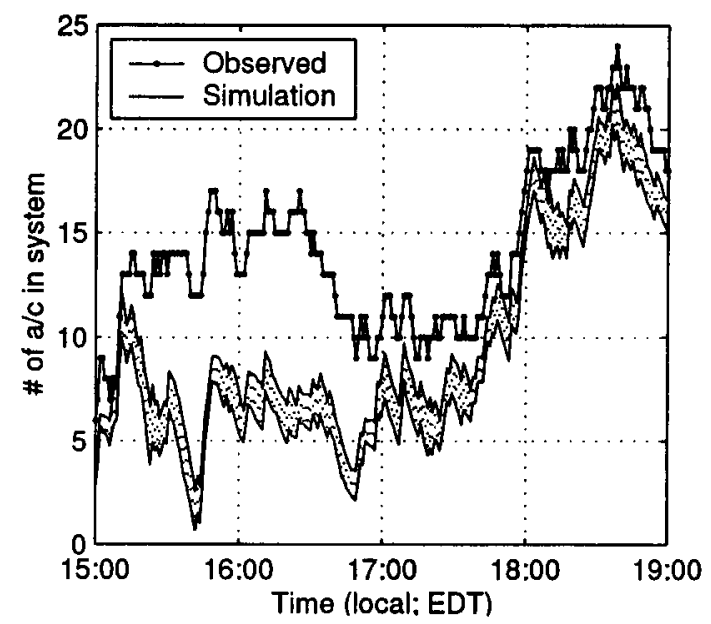

Figure 5: Number of departing a/c (late).

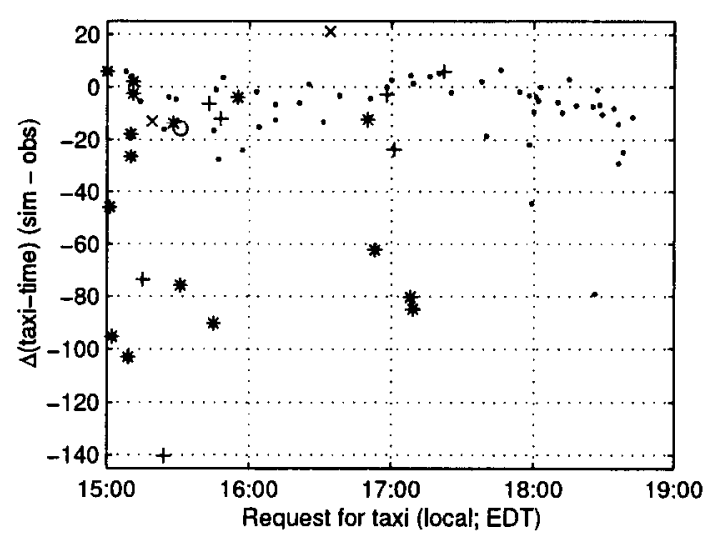

Figure 6: Simulation error (late).

Figure 5 focuses on the period from 15:00 to 19:00, during a period of many more restrictions. The simulation does not accurately track the actual operations from roughly 15:00 to 17:00, but recovers later in the evening.

The simulation errors during the later period are shown in Figure 6. The range of errors is much wider, with flights through ELIOT and PARKE accounting for most of the extreme cases. Both fixes closed from 16:00 to 16:30, and again from 18:10 to 19:00, but had only a 20MIT restriction for the remaining period. Here it is hypothesized that excessive traffic load combined with the fix restrictions to cause the severe observed delays. A threshold effect may have occurred, where delayed traffic was slightly delayed by the spacing requirements, only to be caught by the intermittent closures.

For reference, Figure 7 shows the relationship between the various departure fixes. Note that during the case-study, weather at EWR was mild; the severe departure-fix restrictions were propagated back to EWR from weather-impacted downstream airspace [4]. 


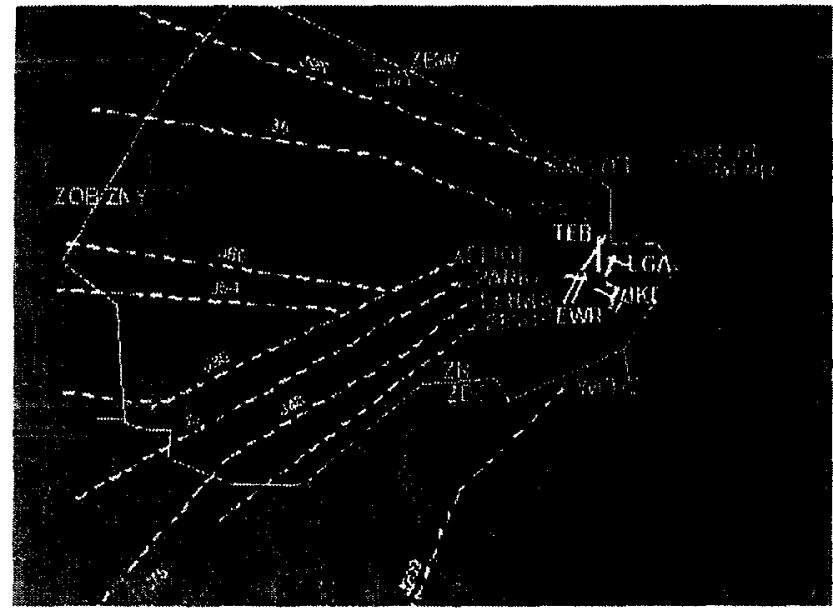

Figure 7: Map of the EWR airspace.

\section{Concluding Remarks}

\subsection{Further Model Improvements}

As seen from the simulation results, the effects of surface traffic congestion and fix closures can be satisfactorily modeled with strikingly simple queueing dynamics. However, departure delays which cannot be explained solely by these effects are also apparent. Severe spacing restrictions can combine with high traffic levels, overloading the flexibility to meet such restrictions via re-sequencing before takeoff or vectoring after takeoff.

To incorporate such effects, the sequencing logic of the tower controllers must be approximated and accurately captured in the model dynamics. One very important issue is to characterize the difficulty of efficient re-sequencing as traffic levels rise and spacing restrictions are made more severe; in particular, a sharp threshold effect would have important consequences for strategic traffic management. Additionally, there is some small degree of flexibility to vector or buffer aircraft after takeoff in the TRACON departure airspace. This effect may be represented as a very small buffering space connected to the runway system (see Figure 8). Work is underway to characterize this effect through interviews with Departure controllers in the TRACON and analysis of TRACON radar flight-tracks. An interesting question concerns the MIT or MINIT spacing requirement which effectively closes a departure fix. It is apparent that some threshold effect must occur between the minimal 3mile in-trail spacing required of all departure aircraft, and the effectively infinite spacing of a fix-closure. It is possible that very large fix-spacing requirements (above the 40MIT sometimes encountered) are not observed because they effectively close the fix under normal departure traffic mixes. It may also be the case that such large spacing requirements are workload-intensive to impose at the runway, and hence their absence from the operational record primarily represents a human-factors limitation.

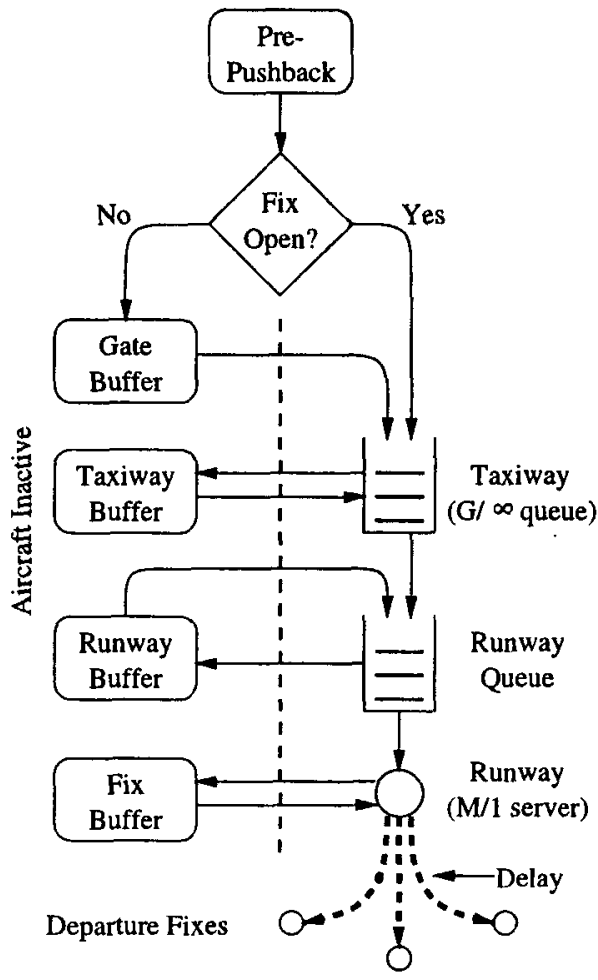

Figure 8: Departure airspace buffering.

\subsection{Applications and "What-If"' Scenarios}

The current Monte Carlo-based model representation is being used to address several open questions raised during the EWR case-study. These questions include the following:

Question: After a fix closure is lifted (or a spacing restriction is relaxed), what is the expected response-time for the actual fix throughput to plateau? Can this response-time be decreased by suitably buffering aircraft at different points in the system?

Question: At many airports, departure fixes are clustered, e.g. the West fixes at EWR. What are the potential benefits and problems of a simple re-routing procedure designed to switch fixes and thus avoid fix closures due to well-localized weather problems?

Question: Assume that some small lead-time is available on upcoming changes to fix restrictions, e.g. the tower controllers learn 10 minutes in advance that a fix closure will be lifted. What are the potential benefits of control strategies which account for this lead-time information? 


\section{References}

[1] K. Andersson, F. Carr, E. Feron, and W. Hall. Analysis and modeling of ground operations at hub airports. In Proceedings of 3rd USA/Europe Air Traffic Management $R \& D$ Seminar, Website: "http://atm-seminar2000. eurocontrol.frr', June 2000. Eurocontrol and the FAA.

[2] G. Andretta, L. Brunetta, A. Odoni, L. Righi, M. Stamatopoulos, and G. Zografos. A set of approximate and compatible models for airport strategic planning on airside and on landside. In Proceedings of 2nd USA/Europe Air Traffic Management R\&D Seminar, Website: "http://atmseminar-98.eurocontrol.frl", December 1998. Eurocontrol and the FAA.

[3] F. Carr. Stochastic modeling and control of airport surface traffic. Master's thesis, Massachusetts Institute of Technology, 2001. Note: these results are also documented (more accessibly) in [1].

[4] A. Evans. Airline and air traffic control response to congestion and delays: A case-study at Newark Airport. Master's thesis, Massachusetts Institute of Technology, 2002 (expected). In progress.

[5] E. Gilbo. Optimizing airport capacity utilization in air traffic flow management subject to constraints at arrival and departure fixes. IEEE Transactions on Control Systems Technology, 5(5):490-503, September 1997.

[6] S. Green. En route spacing tool: Efficient conflictfree spacing to flow-restricted airspace. In Proceedings of 3rd USA/Europe Air Traffic Management R\&D Seminar, Website: "http://atm-seminar-2000.eurocontrol.fr/", June 2000. Eurocontrol and the FAA.

[7] H. Idris. Observations and Analysis of Departure Operations at Boston Logan International Airport. $\mathrm{PhD}$ thesis, Massachusetts Institute of Technology, 2000.

[8] H. Idris, B. Delcaire, I. Anagnostakis, W. Hall, N. Pujet, E. Feron, R. J. Hansman, J. P. Clarke, and A. Odoni. Identification of flow constraints and control points in departure operations at airport systems. Air Traffic Control Quarterly, 2000.

[9] N. Pujet. Modeling and Control of the Departure Process of Congested Airports. PhD thesis, Massachusetts Institute of Technology, 1999. Note: these results are also documented (more accessibly) in [10] and [11].

[10] N. Pujet, B. Delcaire, and E. Feron. Input-output modeling and control of the departure process of busy airports. Air Traffic Control Quarterly, 8(1):1-32, 2000.

[11] N. Pujet and E. Feron. Input-output modeling and control of the departure process of busy airports. In AIAA Conference on Guidance, Navigation and Control, Portland, OR, August 1999. AIAA.

[12] R. Shumsky. Dynamic Statistical Models for the Prediction of Aircraft Take-Off Times. PhD thesis, Massachusetts Institute of Technology, 1995. 